\title{
THE MATHERON REPRESENTATION THEOREM FOR GRAY-SCALE MORPHOLOGICAL FILTERS
}

\author{
G. CROMBEZ
}

(Communicated by Andrew Odlyzko)

\begin{abstract}
We present a new proof of the Matheron representation theorem for gray-scale morphological filters, without using either the representation theorem for subsets of the plane or the umbra transform.
\end{abstract}

\section{INTRODUCTION}

An important theorem in mathematical morphology is the Matheron representation theorem, which for subsets of $R^{2}$ may be stated as follows [2, Chapter 5]: if $\Psi$ is an increasing translation-invariant mapping between subsets of $R^{2}$, then for any subset $A$ of $R^{2}$ we have

$$
\Psi(A)=\bigcup_{B \in \operatorname{Ker} \Psi} \mathscr{E}(A, B),
$$

where $\mathscr{E}(A, B)$ denotes the erosion of the set $A$ by the set $B$, and $\operatorname{Ker} \Psi$ (the kernel of $\Psi$ ) is the collection of all subsets $B$ of $R^{2}$ such that $\Psi(B)$ contains the origin.

In [2, Chapter 7], the Matheron representation theorem has been extended to the case of gray-scale morphological filters. In their proof, however, the authors use the representation theorem for subsets of $R^{2}$ and also the so-called "umbra transform". In the following sections, we give a proof of the Matheron representation theorem for gray-scale morphological filters without using either the theorem for $R^{2}$ or the umbra transform; moreover, the theorem for subsets of $R^{2}$ is just a special case of our general theorem.

\section{Definitions AND NOtATIONS}

Let $G$ be a (not necessarily commutative) group with a multiplicative group operation and identity $e$; the inverse of $x \in G$ is denoted by $x^{-1}$.

Received by the editors July 19, 1988.

1980 Mathematics Subject Classification (1985 Revision). Primary 94A12, 68V70; Secondary $52 \mathrm{~A} 22$.

Key words and phrases. Mathematical morphology, image analysis, translation invariant mapping. 
When $V$ is a subgroup of the additive group $R$ of real numbers, $G \times V$ is also a group for the following operation: for $x \in G, y \in G, r \in V$, and $s \in V$, we define

$$
(x, r) \cdot(y, s)=(x y, r+s) .
$$

For the case of mathematical morphology, $G$ may be thought of as $\left(R^{2},+\right)$ or $\left(Z^{2},+\right)$, while for $V$ we take $V=R, V=Z$ or $V=\{0\}$, where $Z$ is the set of integers. For $V=\{0\}, G \times V$ can be identified in the usual manner with $G$.

We denote by $\mathscr{S}$ the set of bounded functions $f$ defined on a subset $D_{f}$ (also written as $D(f))$ of $G$ and with values in $V \cdot \mathscr{S}^{*}$ is the set of functions defined on or subset of $G$ and with values in $V \cup\{+\infty\}$. We identify $f$ with its graph $G(f)$; hence, for $f \in \mathscr{S}$ we have $f \equiv G(f)=\left\{(x, f(x)): x \in D_{f}\right\}$, which is a subset of $G \times V$. When $V=\{0\}$, then $f=\left\{(x, 0): x \in D_{f}\right\} \equiv D_{f}$, which shows that in that case we may consider $\mathscr{S}$ to be the set $2^{G}$.

For $f$ and $g$ in $\mathscr{S}$, the notation $f \ll g$ means that $D_{f} \subset D_{g}$ and $f(x) \leqq$ $g(x) \forall x \in D_{f}$; specifically, for $V=\{0\}$, we have $f \ll g \Leftrightarrow D_{f} \subset D_{g}$. When $(b, r) \in G \times V$ and $f \in \mathscr{S}$, the left-translate ${ }_{(b, r)} f$ is defined by $\left.{ }_{(b, r)} f\right)(x)=$ $f\left(b^{-1} x\right)+r$. In particular, for $V=\{0\}$, we have ${ }_{(b, 0)} f \equiv D_{(b, 0)} f=(b, 0) \cdot D_{f}$, where in the last term we identify $D_{f}$ with $\left(D_{f}, 0\right)$.

According to the terminology in [2], a mapping $\Psi: \mathscr{S} \rightarrow \mathscr{S}^{*}$ is called increasing if $f \ll g$ implies $\Psi(f) \ll \Psi(g)$ for all $f, g \in \mathscr{S} ; \Psi$ is called left translation invariant if $\Psi\left(_{(b, r)} f\right)={ }_{(b, r)} \Psi(f)$ for all $(b, r) \in G \times V$ and all $f \in \mathscr{S}$. An increasing left translation invariant mapping $\Psi: \mathscr{S} \rightarrow \mathscr{S}^{*}$ is called a morphological filter. The kernel $\operatorname{Ker} \Psi$ of such filter is defined by $\operatorname{Ker} \Psi=$ $\{f \in \mathscr{S}: \Psi(f)(e) \geqq 0\}$. For $V=\{0\}$, this leads to $\operatorname{Ker} \Psi=\left\{D_{f}: e \in \Psi\left(D_{f}\right)\right\}$, which corresponds to the usual definition for mappings between subsets of $R^{2}$. The final notation is the erosion $\mathscr{E}(f, g)$ of a function $f$ in $\mathscr{S}$ by a function $g$ in $\mathscr{S}$; again, it is a function in $\mathscr{S}$ defined as

$$
\mathscr{E}(f, g)=\left\{(x, t) \in G \times V: x D_{g} \subset D_{f}, t=\sup \left\{s \in V: g\left(x^{-1} \cdot\right)+s \leqq f(\cdot)\right\}\right.
$$

This definition may be found in [2], and also in [1], where we gave a unifying theory for the morphological operations dilation, erosion and opening for grayscale images; in particular, for $V=\{0\}$ and $G=R^{2}$, we are again led to the erosion of two subsets of $R^{2}$. The only properties we need in the sequel, and which may readily be derived from the definition, are

$$
\begin{gathered}
\mathscr{E}\left(_{(h, r)} f, g\right)=_{(b, r)} \mathscr{E}(f, g), \\
f_{1} \ll f_{2} \Rightarrow \mathscr{E}\left(f_{1}, g\right) \ll \mathscr{E}\left(f_{2}, g\right) .
\end{gathered}
$$




\section{The MATHERON REPRESENTATION THEOREM}

Proposition 1. Let $\Psi_{1}$ and $\Psi_{2}$ be morphological filters. Then

$$
\operatorname{Ker} \Psi_{1} \subset \operatorname{Ker} \Psi_{2} \Leftrightarrow \Psi_{1}(f) \ll \Psi_{2}(f), \quad \forall f \in \mathscr{S} \text {. }
$$

Proof. Suppose $\Psi_{1}(f) \ll \Psi_{2}(f)$ for all $f \in \mathscr{S}$. Given $f \in \operatorname{Ker} \Psi_{1}$, then $\Psi_{1}(f)(e) \geqq 0$. From our assumption we have $D\left(\Psi_{1}(f)\right) \subset D\left(\Psi_{2}(f)\right)$ and $\Psi_{1}(f)(x) \leqq \Psi_{2}(f)(x)$ for all $x \in D\left(\Psi_{1}(f)\right)$. Hence, it follows that $\Psi_{2}(f)(e)$ is defined and $\Psi_{1}(f)(e) \leqq \Psi_{2}(f)(e)$; therefore $f \in \operatorname{Ker} \Psi_{2}$.

Conversely, suppose $\operatorname{Ker} \Psi_{1} \subset \operatorname{Ker} \Psi_{2}$. We must show that for each $f$ in $\mathscr{S}, D\left(\Psi_{1}(f)\right) \subset D\left(\Psi_{2}(f)\right)$, and for each $x$ in $D\left(\Psi_{1}(f)\right), \Psi_{1}(f)(x) \leqq$ $\Psi_{2}(f)(x)$.

(i) If it is not true that $D\left(\Psi_{1}(f)\right) \subset D\left(\Psi_{2}(f)\right)$ for all $f$ in $\mathscr{S}$, then there exists $f \in \mathscr{S}$ and $x \in D\left(\Psi_{1}(f)\right)$ such that $x \notin D\left(\Psi_{2}(f)\right)$. Suppose $\Psi_{1}(f)(x)=a \in V$, and consider the function ${ }_{\left(x^{-1},-a\right)} f$. Then

$$
\Psi_{1}\left(_{\left(x^{-1},-a\right)} f\right)(e)=\Psi_{1}(f)(x)-a=0,
$$

which means that ${ }_{\left(x^{-1},-a\right)} f$ belongs to $\operatorname{Ker} \Psi_{1}$; however, $\Psi_{2}\left({ }_{\left(x^{-1},-a\right)} f\right)(e)$ is not defined since $x \notin D\left(\Psi_{2}(f)\right)$, and so ${ }_{\left(x^{-1},-a\right)} f$ is not an element of $\operatorname{Ker} \Psi_{2}$. This is a contradiction.

(ii) Suppose there exists $f \in \mathscr{S}$ and $x \in D\left(\Psi_{1}(f)\right)$ such that $\Psi_{1}(f)(x)>$ $\Psi_{2}(f)(x)$. If $\Psi_{1}(f)(x)=a \in V$, consider again the function ${ }_{\left(x^{-1},-a\right)} f$; then $\Psi_{1}\left({ }_{\left(x^{-1},-a\right)} f\right)(e)=0$, while $\Psi_{2}\left(_{\left(x^{-1},-a\right)} f\right)(e)<0$. This is again a contradiction.

(When $\Psi_{1}(f)(u)=+\infty$ in (i) or (ii), the proof is easily adapted).

Corollary 1. When $\Psi_{1}$ and $\Psi_{2}$ are morphological filters, then

$$
\Psi_{1}=\Psi_{2} \Leftrightarrow \operatorname{Ker} \Psi_{1}=\operatorname{Ker} \Psi_{2} \text {. }
$$

Given a fixed function $g$ in $\mathscr{S}$, we define the mapping $\Psi_{g}$ on $\mathscr{S}$ by means of

$$
\Psi_{g}(f)=\mathscr{E}(f, g), \quad f \in \mathscr{S} \text {. }
$$

Proposition 2. (i) $\Psi_{g}$ is a morphological filter.

(ii) $\operatorname{Ker} \Psi_{g}=\{f: g \ll f\}$.

Proof. (i) This follows immediately from the properties of erosion, as mentioned at the end of $\S 2$.

(ii) $f \in \operatorname{Ker}_{g}$ iff $\Psi_{g}(f)(e) \geqq 0$ iff $\mathscr{E}(f, g)(e) \geqq 0$.

Now $e \in D(\mathscr{E}(f, g))$ iff $e D_{g} \subset D_{f}$, which is fulfilled as soon as $g \ll f$. Also $\mathscr{E}(f, g)(e) \geqq 0$ iff $\sup \left\{s \in V: s \leqq f(z)-g(e z), \forall z \in D_{g}\right\} \geqq 0$, which is true iff $g(z) \leqq f(z), \forall z \in D_{g}$.

Lemma 1. Let $\Psi$ be a morphological filter. Let $\Psi_{1}$ be the mapping $\mathscr{S} \rightarrow \mathscr{S}^{*}$, defined as

$$
D\left(\Psi_{1}(f)\right)=\bigcup_{g \in \operatorname{Ker} \Psi} D(\mathscr{E}(f, g))
$$

$\left(\Psi_{1}(f)\right)(x)=\sup \mathscr{E}\{(f, g)(x): g \in \operatorname{Ker} \Psi$ such that $x \in D(\mathscr{E}(f, g))\}$.

Then $\Psi_{1}$ is a morphological filter. 
Proof. (i) We first investigate the left translation invariance of $\Psi_{1}$. Let $(b, r) \in$ $G \times V, f \in \mathscr{S}$. Then, since $D\left(_{(b, r)} f\right)=b D_{f}$ (for the group operation in $G$ ), we have

$$
\begin{aligned}
D\left(\Psi_{1}\left(_{(b, r)} f\right)\right. & =\bigcup_{g \in \operatorname{Ker} \Psi} D\left(\mathscr{E}\left(_{(b, r)} f, g\right)\right) \\
& =\bigcup_{g \in \operatorname{Ker} \Psi} D\left(\left(_{(b, r)} \mathscr{E}(f, g)\right)\right. \\
& =\bigcup_{g \in \operatorname{Ker} \Psi} b D(\mathscr{E}(f, g)),
\end{aligned}
$$

and also

$$
D\left({ }_{(b, r)} \Psi_{1}(f)\right)=b D\left(\Psi_{1}(f)\right)=\bigcup_{g \in \operatorname{Ker} \Psi} b D(\mathscr{E}(f, g)) .
$$

Moreover,

$$
\begin{aligned}
\Psi_{1}\left(_{(b, r)} f\right)(x) & =\sup \left\{\mathscr{E}\left(_{(\mathrm{b}, \mathrm{r})} \mathrm{f}, \mathrm{g}\right)(\mathrm{x}): g \in \operatorname{Ker} \Psi \text { such that } x \in D\left(\mathscr{E}\left(_{(b, r)} f, g\right)\right)\right\} \\
& =\sup \left\{{ }_{(\mathrm{b}, \mathrm{r})} \mathscr{E}(\mathrm{f}, \mathrm{g})(\mathrm{x}): g \in \operatorname{Ker} \Psi \text { such that } x \in b D(\mathscr{E}(f, g))\right\} \\
& =\sup \left\{\left(\mathscr{E}(\mathbf{f}, \mathbf{g})\left(\mathrm{b}^{-1} \mathrm{x}\right)+\mathrm{r}\right): g \in \operatorname{Ker} \Psi \text { such that } b^{-1} x \in D(\mathscr{E}(f, g))\right\},
\end{aligned}
$$

which is exactly the value of $(b, r) \Psi_{1}(f)(x)$.

(ii) To show that $\Psi_{1}$ is also increasing, we have to prove that $f \ll h$ implies $D\left(\Psi_{1}(f)\right) \subset D\left(\Psi_{1}(h)\right)$ and $\Psi_{1}(f)(x) \leqq \Psi_{1}(h)(x)$ for all $x \in D\left(\Psi_{1}(f)\right)$.

This is almost obvious from the definition of $\Psi_{1}$, due to the fact that $D(\mathscr{E}(f, g)) \subset D(\mathscr{E}(h, g))$ and that $\mathscr{E}(f, g)(x) \leq \mathscr{E}(h, g)(x)$.

Theorem 1. The Matheron representation theorem. Let $\Psi$ be a morphological filter. Then for each $f$ in $\mathscr{S}, \Psi(f)$ is the function defined as

$$
D(\Psi(f))=\bigcup_{g \in \operatorname{Ker} \Psi} D(\mathscr{E}(f, g)), \text { and }
$$

$\Psi(f)(x)=\sup \{\mathscr{E}(\mathrm{f}, \mathrm{g})(\mathrm{x}): g \in \operatorname{Ker} \Psi$ such that $x \in D(\mathscr{E}(f, g))\}$.

Proof. We first remark that, for $g \in \operatorname{Ker} \Psi$ and $g \ll h, h \in \operatorname{Ker} \Psi$ also. Hence, $\operatorname{Ker} \Psi \supset \bigcup_{g \in \operatorname{Ker} \Psi}\{h: g \ll h\}$. But it is trivial that any $g$ in $\operatorname{Ker} \Psi$ also belongs to the set $\{h: g \ll h\}$. This leads to

$$
\operatorname{Ker} \Psi=\bigcup_{g \in \operatorname{Ker} \Psi}\{h: g \ll h\} .
$$

Taking into account the function $\Psi_{1}$ introduced in Lemma 1, the theorem will be proved if, according to Corollary 1, we show that $\operatorname{Ker} \Psi=\operatorname{Ker} \Psi_{1}$.

First, suppose that $f \in \operatorname{Ker} \Psi_{1}$; then $e \in D\left(\Psi_{1}(f)\right)$ and $\Psi_{1}(f)(e) \geqq 0$. From the first conclusion and the definition of $\Psi_{1}$, we derive that there exists $g \in \operatorname{Ker} \Psi$ such that $e \in D(\mathscr{E}(f, g))$, or, to put it another way, there exists $g \in \operatorname{Ker} \Psi$ such that $D_{g} \subset D_{f}$. The second conclusion leads to

$$
\sup \left\{\mathscr{E}(\mathrm{f}, \mathrm{g})(\mathrm{e}) \geqq 0: g \in \operatorname{Ker} \Psi \text { such that } D_{g} \subset D_{f}\right\}
$$


which may also be written as

$$
\sup \left\{\left(\sup \{\mathrm{s}: \mathrm{s} \leqq \mathrm{f}(\mathrm{z})-\mathrm{g}(\mathrm{z})\}: g \in \operatorname{Ker} \Psi \text { such that } D_{g} \subset D_{f}\right\} \geqq 0\right. \text {. }
$$

From this we immediately conclude that there exists $g \in \operatorname{Ker} \Psi$ with $D_{g} \subset D_{f}$ and $g(z) \leqq f(z), \forall z \in D_{g}$, which means that $f \in \operatorname{Ker} \Psi$; hence $\operatorname{Ker} \Psi_{1} \subset$ $\operatorname{Ker} \Psi$.

Conversely, suppose that $f \in \operatorname{Ker} \Psi$; then there exists $g \in \operatorname{Ker} \Psi$ such that $D_{g} \subset D_{f}$ and $g(z) \leqq f(z), \forall z \in D_{g}$. In order for $f \in \operatorname{Ker} \Psi_{1}$ we have to show that $e \in D\left(\Psi_{1}(f)\right)$ and $\Psi_{1}(f)(e) \geqq 0$.

Now, from Lemma $1, e \in D\left(\Psi_{1}(f)\right)$ iff there exists $g \in \operatorname{Ker} \Psi$ such that $e \in D(\mathscr{E}(f, g))$, which is true since $D_{g} \subset D_{f}$ for some $g \in \operatorname{Ker} \Psi$.

Again from Lemma $1, \Psi_{1}(f)(e)=\sup \left\{\mathscr{E}(\mathrm{f}, \mathrm{g})(\mathrm{e}): g \in \operatorname{Ker} \Psi\right.$ such that $D_{g}$ $\left.\subset D_{f}\right\}$, with $\mathscr{E}(f, g)(e)=\sup \{s \in V: s \leqq f(z)-g(z)\}$, and this is already non-negative for one particular $g$; hence $\Psi_{1}(f)(e) \geqq 0$. So we also have $\operatorname{Ker} \Psi \subset \operatorname{Ker} \Psi_{1}$. According to Corollary $1, \Psi=\Psi_{1}$, which proves the theorem.

We finally remark that, when taking in Theorem $1 V=\{0\}$ and $G=$ $\left(R^{2},+\right)$, we obtain as a special case the Matheron representation theorem for subsets $R^{2}$.

\section{REFERENCES}

1. G. Crombez, Group theoretical methods in gray-scale mathematical morphology, (preprint).

2. C. R. Giardina and E. R. Dougherty, Morphological methods in image and signal processing, Prentice Hall, Englewood Cliffs, New Jersey, 1988.

3. G. Matheron, Random sets and integral geometry, John Wiley and Sons, New York, 1975.

4. J. Serra, Image analysis and mathematical morphology, Academic Press, London, 1982.

State University of Ghent, Seminar of Higher Analysis, Krijgslaan 281, B-9000, Gent BELGIUM 